November 20, 2018 0:2 WSPC/INSTRUCTION FILE 1dcorre

International Journal of Modern Physics B

(C) World Scientific Publishing Company

\title{
EFFECT OF DISORDER ON THE INTERACTING FERMI GASES IN A ONE-DIMENSIONAL OPTICAL LATTICE
}

\author{
GAO XIANLONG \\ Department of Physics, Zhejiang Normal University, Jinhua, Zhejiang Province, 321004, China \\ M. POLINI and M. P. TOSI \\ NEST-CNR-INFM and Scuola Normale Superiore, I-56126 Pisa, Italy \\ B. TANATAR* \\ Department of Physics, Bilkent University, Ankara, 06800, Turkey \\ *E-mail: tanatar@fen.bilkent.edu.tr \\ Received Day Month Year \\ Revised Day Month Year
}

\begin{abstract}
Interacting two-component Fermi gases loaded in a one-dimensional (1D) lattice and subjected to an harmonic trapping potential exhibit interesting compound phases in which fluid regions coexist with local Mott-insulator and/or band-insulator regions. Motivated by experiments on cold atoms inside disordered optical lattices, we present a theoretical study of the effects of a correlated random potential on these ground-state phases. We employ a lattice version of density-functional theory within the local-density approximation to determine the density distribution of fermions in these phases. The exchange-correlation potential is obtained from the Lieb-Wu exact solution of FermiHubbard model. On-site disorder (with and without Gaussian correlations) and harmonic trap are treated as external potentials. We find that disorder has two main effects: (i) it destroys the local insulating regions if it is sufficiently strong compared with the on-site atom-atom repulsion, and (ii) it induces an anomaly in the inverse compressibility at low density from quenching of percolation. For sufficiently large disorder correlation length the enhancement in the inverse compressibility diminishes.
\end{abstract}

Keywords: Fermi-Hubbard model; optical lattices; disorder.

\section{Introduction}

Disorder and interaction effects in condensed matter systems have a long and rich history. The interplay between them has been the subject of continuing interest. The notable examples range from two-dimensional electron systems with long-ranged Coulomb interactions 1 to liquid ${ }^{4} \mathrm{He}$ absorbed in various substances such as aerogel and vycor ${ }^{2}$ and granular superconductors $\frac{3}{3}$ In the former system experimental and theoretical investigations 4 reveal the crucial role played by disorder as the twodimensional electron system undergoes a metal-insulator transition. Furthermore, thermodynamic quantities carry the signature of phase transition $\stackrel{516}{ }$ along with 
transport properties.

In recent years, cold atomic systems are being used to investigate the interplay between single-particle randomness and interaction effects with the help of optical lattices allowing access to strong coupling regimes through the depression of kinetic energy! 9 Furthermore, the on-site interaction can be tuned either indirectly by changing the strength of the lasers that create the optical lattice or directly by means of a Feshbach resonance 12

After the initial observation of superfluid to Mott insulator transition using bosonic atoms in an optical lattice 10 and creation of disorder potentials, $\frac{11}{11}$ studies on fermionic atoms 1213 are beginning to be explored. A couple of forthcoming reviews 14 encompass many aspects of the theory of ultracold Fermi gases.

In this work we study the interaction and disorder effects on a one-dimensional, two-component Fermi gas trapped in a harmonic confinement potential and an optical lattice. We use the lattice version of density functional theory $\frac{15}{15}$ taking advantage of the exact solution of the one-dimensional Fermi-Hubbard model 16 Ground-state calculations in the absence of disorder have been performed within a number of numerical techniques $\overline{17 / 18}$ to identify various phases. In the present approach, the harmonic confinement potential and the disorder potential are treated as part of the Kohn-Sham potential within a local-density approximation which has an exact representation for the uniform case.

Generalizing our earlier work 19 on the effects of uncorrelated disorder, we here study correlated disorder which depends on the correlation length as a parameter. The site occupation profiles and thermodynamic stiffness are determined to study the effects of disorder. Our motivation for considering correlated disorder comes from the experiments 7 on Bose-Einstein condensates in optical speckle potentials where it was noted that the correlation length is several times longer than the lattice spacing.

The paper is organized as follows. In Sec. 2 we briefly outline the Fermi-Hubbard model in a harmonic potential and disorder. We then give the main ingredients of our density-functional theory approach. Section 3 presents our results on site occupations in the presence of correlated disorder. We conclude with a brief summary in Sec. 4.

\section{Theory and Model}

We consider a two-component Fermi gas with $N$ atoms constrained to move under harmonic confinement of strength $V_{2}$ inside a disordered $1 D$ optical lattice with unit lattice constant and $L$ lattice sites $i \in[1, L]$. The system is described by a single-band Hubbard Hamiltonian,

$$
\hat{\mathcal{H}}=-t \sum_{i=1}^{L-1} \sum_{\sigma}\left(\hat{c}_{i \sigma}^{\dagger} \hat{c}_{i+1 \sigma}+\text { H.c. }\right)+U \sum_{i=1}^{L} \hat{n}_{i \uparrow} \hat{n}_{i \downarrow}
$$




$$
+V_{2} \sum_{i=1}^{L}(i-L / 2)^{2} \hat{n}_{i}+\sum_{i=1}^{L} \varepsilon_{i} \hat{n}_{i} .
$$

Here $t_{i j}=t>0$ if $i, j$ are nearest sites and zero otherwise, $\sigma=\uparrow, \downarrow$ is a pseudospin$1 / 2$ label for two internal hyperfine states, $\hat{n}_{i \sigma}=\hat{c}_{i \sigma}^{\dagger} \hat{c}_{i \sigma}$ is the pseudospin-resolved site occupation operator, and $\hat{n}_{i}=\sum_{\sigma} \hat{n}_{i \sigma}$. The effect of disorder is simulated by the last term in Eq. (11). The above Hamiltonian without the confining potential and disorder term has been solved exactly using the Bethe Ansatz technique by Lieb and $\mathrm{Wu} \frac{16}{16}$ The exact solution provides us with the ground-state energy at any coupling strength $u=U / t$ and filling $n=N / L$. In the noninteracting and unconfined limit (i.e., without the $U$ and trap terms) one recovers the Anderson mode $\sqrt{20}$ studied intensively for the Anderson localization problem. The successful creation of optical lattices to study cold atomic systems has led to renewed interest in these model systems, since the atomic systems offer a wide range of control on the various parameters.

In this work we generalize our previous study $\underline{19}$ on uncorrelated noise to Gaussian correlated disorder, defined as

$$
\varepsilon_{i}=\varepsilon_{i}(\xi)=\frac{1}{\sqrt{2 \pi \xi}} \sum_{j=1}^{L} \exp \left[-\frac{(i-j)^{2}}{2 \xi}\right] \mathcal{W}_{j},
$$

where $\xi$ is the correlation length (in units of the lattice spacing, which is set to unity throughout this work) and $\mathcal{W}_{j}$ is randomly chosen at each site $j$ from a uniform distribution in the range $[-W / 2, W / 2]$. Because of the following mathematical identity,

$$
\lim _{\xi \rightarrow 0} \frac{1}{\sqrt{2 \pi \xi}} \exp \left[-\frac{(i-j)^{2}}{2 \xi}\right]=\delta_{i j}
$$

Eq. (2) tends smoothly to uncorrelated (i.e. white) noise in the limit $\xi \rightarrow 0$. We illustrate typical behavior of $\varepsilon_{i}$ over a lattice of $L=200$ sites in Fig. 1 for some values of $\xi$. As the correlation length increases the correlated disorder potential becomes smoother with amplitude smaller than that in the uncorrelated case.

A particular set of values $\varepsilon_{i}$ is a realization of disorder. Each realization defines an external potential $\mathcal{V}_{i}=V_{2}\left(i-N_{s} / 2\right)^{2}+\varepsilon_{i}$ which is the sum of a harmonic trap potential and disorder. the site-occupation functional theory 15 (SOFT) to determine the site occupation $n_{i}=\left\langle\Psi\left|\hat{n}_{i}\right| \Psi\right\rangle$ where $\Psi$ is the ground-state of $\mathcal{H}$ for this particular disorder realization. The site occupation $\mathcal{N}_{i}$ is obtained by the disorder ensemble average, $\mathcal{N}_{i}=\left\langle\left\langle n_{i}\right\rangle\right\rangle_{\text {dis. }}$. SOFT is the discrete or lattice version of the density functional theory which has been successfully applied to the present system in the absence 18 and presence 19 of uncorrelated disorder. In the clean limit the localdensity approximation which we adopt has been shown to be reliable through extensive comparisons with accurate quantum Monte Carlo calculations 18 . Local-density approximation based density-functional schemes for disordered systems have been employed to study the low-density compressibility anomaly in the two-dimensional 
4 Gao et al.

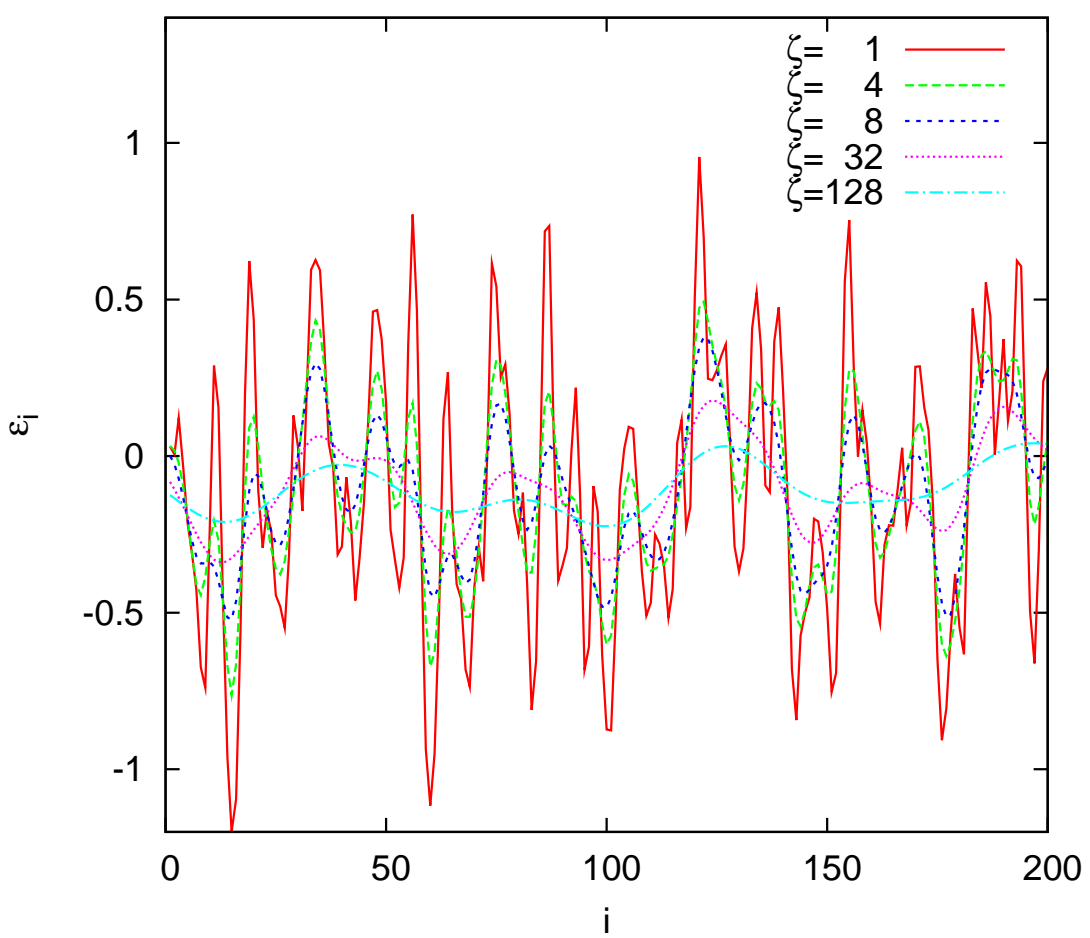

Fig. 1. (color online) The correlated disorder potential $\varepsilon_{i}$ as a function of the site position for a lattice of $L=200$ sites. The disorder strength is $W / t=3$. Different values of $\xi$ are indicated in the legend.

metal-insulator transition (see Ref. 6, 21]) and the statistical properties of $2 D$ disordered quantum dots.22

The total energy is a unique functional of $n_{i}$ which can be written as

$$
\mathcal{E}[n]=\sum_{i} \epsilon(n, u)+\sum_{i} \mathcal{V}_{i}\left(z_{i}\right) n\left(z_{i}\right),
$$

where $\epsilon(n, u)$ is the ground-state energy of the Hubbard Hamiltonian as obtained by Lieb and $\mathrm{Wu}[16$ The Euler-Lagrange equation that follows from the above energy density is

$$
\frac{\partial \epsilon}{\partial n}+v_{\mathrm{KS}}\left(z_{i}\right)=\text { constant }
$$

where the Kohn-Sham potential is

$$
v_{\mathrm{KS}}=\frac{1}{2} U n+v_{\mathrm{xc}}\left(z_{i}\right)+\mathcal{V}_{i}\left(z_{i}\right)
$$

Within the local-density approximation, exchange-correlation potential is approxi- 
mated by

$$
v_{\mathrm{xc}}=\frac{\partial}{\partial n}\left[\epsilon(n, u)-\epsilon(n, 0)-\frac{1}{4} U n^{2}\right] .
$$

The above set of equations allow us to obtain the site occupations $n_{i}$ and their disorder averages $\mathcal{N}_{i}$.

\section{Results and Discussion}

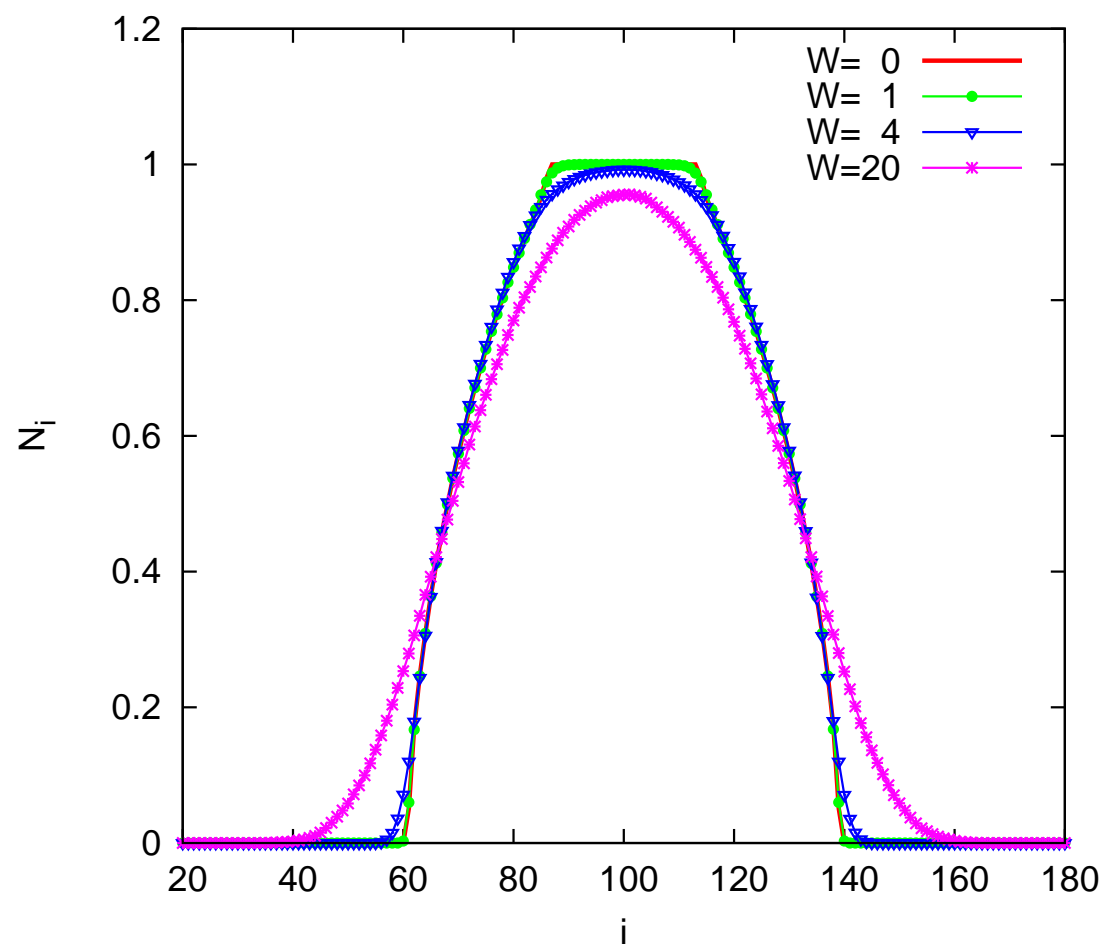

Fig. 2. (color online) Site occupation $\mathcal{N}_{i}$ as a function of site position $i$ for $N=60$ fermions with $u=4, \xi=4$, and $V_{2} / t=2.5 \times 10^{-3}$ in a lattice with $L=200$ sites. The four curves have been calculated for different values of disorder strength: $W / t=0$ (solid line), $W / t=1$ (circles), $W / t=4$ (triangles), and $W / t=20$ (squares).

Before we describe the effects of disorder on the trapped lattice fermions, we outline the various ground-state phases of a clean system as obtained by previous numerical studies $\frac{18}{18}$ There are altogether five phases $(\mathcal{A} \ldots \mathcal{E})$ controlled by the interaction strength $U / t$, number of fermions $N$, and number of lattice sites $L$. Phase $\mathcal{A}$ is a fluid with $0<n_{i}<2$. In phase $\mathcal{B}$ a Mott insulated occupies the central region of the trap with $n_{i}=1$. In phase $\mathcal{C}$ a fluid with $1<n_{i}<2$ is embedded in the 
Mott plateau. Phase $\mathcal{D}$ is a band insulator with $n_{i}=2$ surrounded by fluid edges and embedded in the Mott plateau. Finally, in phase $E$ a band insulator in the central region of the trap coexists with fluid edges. The sketch of site occupations in these phases was given in Ref.19.

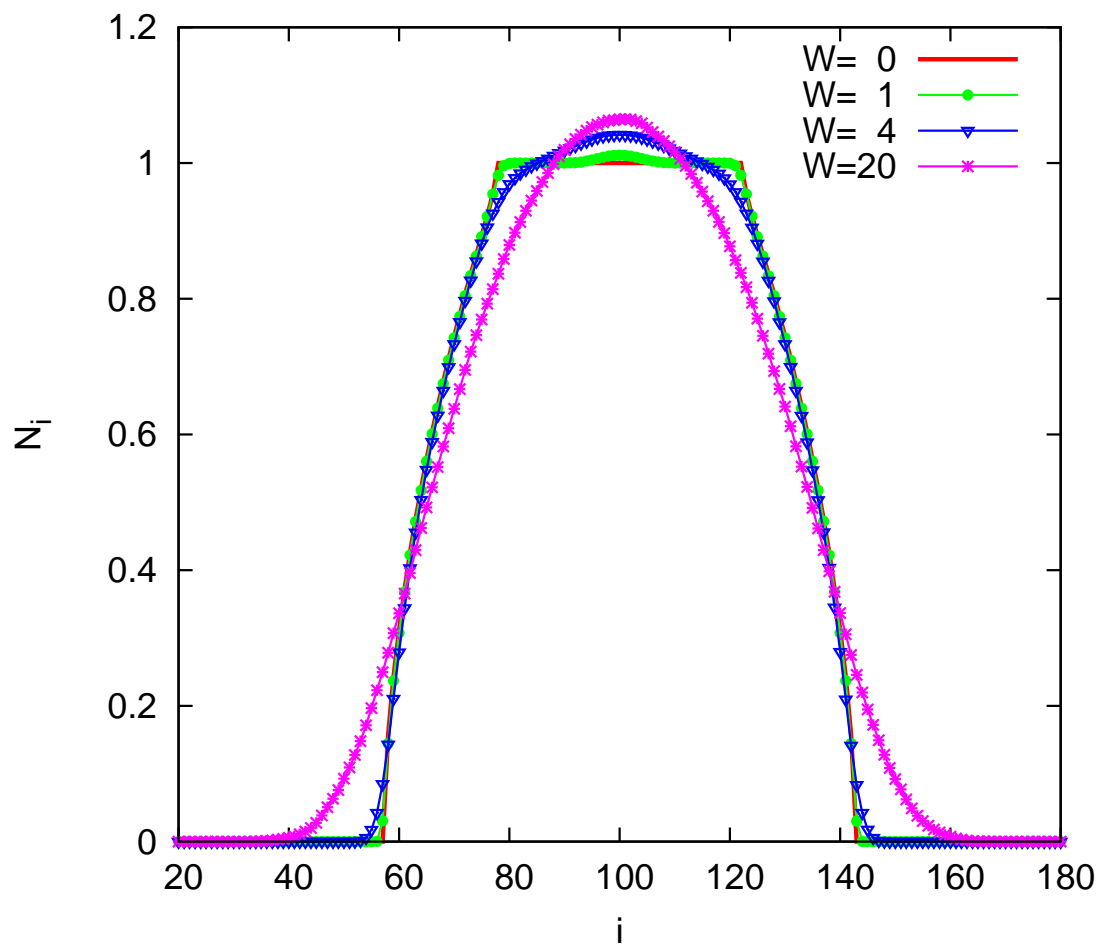

Fig. 3. (color online) Site occupation $\mathcal{N}_{i}$ as a function of site position $i$ for $N=70$ fermions with $u=4, \xi=4$, and $V_{2} / t=2.5 \times 10^{-3}$ in a lattice with $L=200$ sites. The four curves have been calculated for different values of disorder strength: $W / t=0$ (solid line), $W / t=1$ (circles), $W / t=4$ (triangles), and $W / t=20$ (squares).

In Fig. 2 we show the disorder-averaged site occupation $\mathcal{N}_{i}$ for a system of fermions with $N=60$ in an optical lattice with $L=200$ sites. The interaction strength is $u=4$ and the trap potential is $V_{2} / t=1.5 \times 10^{-3}$. Here we fixed the disorder correlation length to be $\xi=4$ and varied the disorder strength $W / t$. The clean system is in phase $\mathcal{B}$. As in the case of uncorrelated disorder 19 we find that with increasing $W / t$ the Mott insulating region is depleted and the site occupation profile $\mathcal{N}_{i}$ broadens. However, the rate of depletion and broadening are smaller than the uncorrelated disorder. In other words, the Mott insulating region is more stable against the formation of a disordered fluid phase in the presence of correlated disorder. 


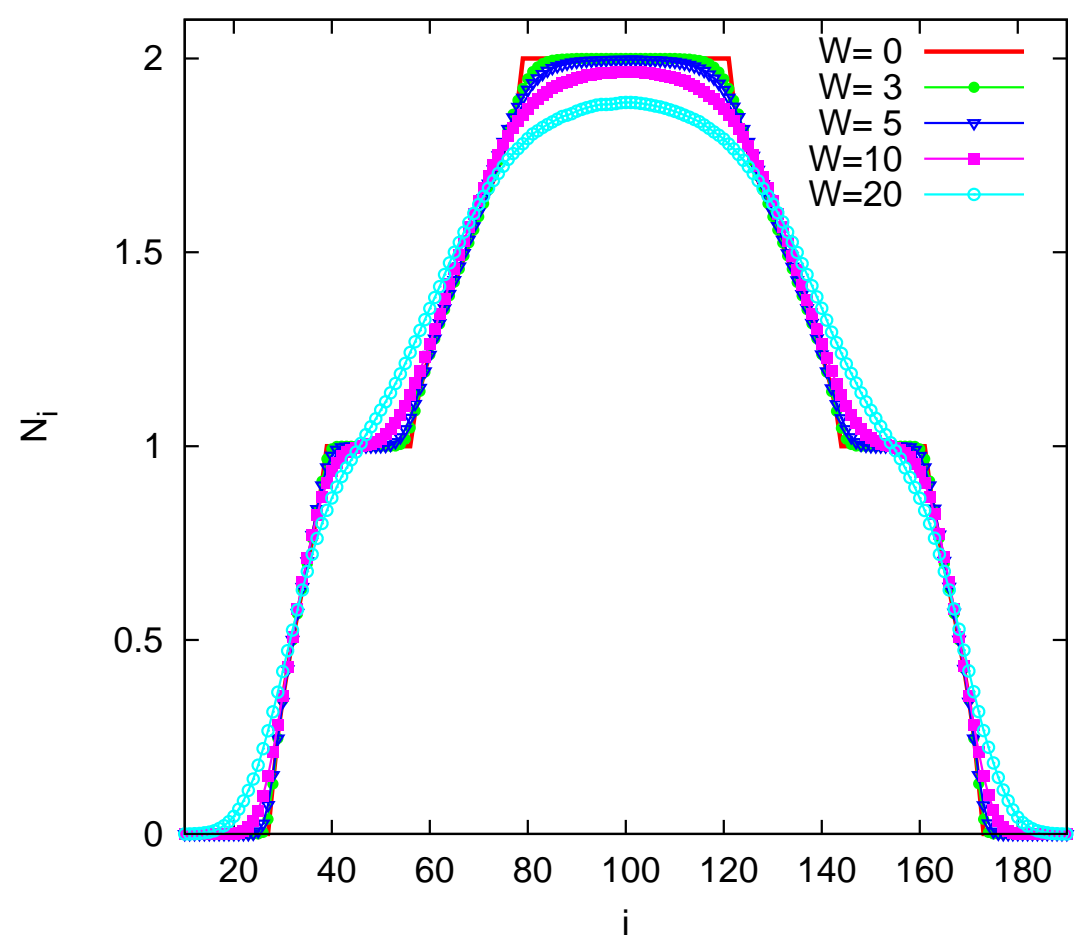

Fig. 4. (color online) Site occupation $\mathcal{N}_{i}$ as a function of site position $i$ for $N=200$ fermions with $u=8, \xi=4$, and $V_{2} / t=2.5 \times 10^{-3}$ in a lattice with $L=200$ sites. The five curves have been calculated for different values of disorder strength: $W / t=0$ (solid line), $W / t=3$ (solid circles), $W / t=5$ (triangles), $W / t=10$ (squares), and $W / t=20$ (empty circles).

In Fig. 3 we display the disorder averaged site occupation $\mathcal{N}_{i}$ for a system with $N=70$ atoms keeping the rest of the parameters the same as in Fig. 2. $N=70$ is the critical number of atoms at which the phase transition $\mathcal{B} \rightarrow \mathcal{C}$ occurs in the clean limit. For weak uncorrelated disorder a fluid phase with $\mathcal{N}_{i}>1$ is induced at the center of the trap. Essentially the same behavior is observed for correlated disorder $(\xi=4)$. Sufficiently strong $W / t$ destroys the Mott plateau completely and the system becomes a disordered fluid with $\mathcal{N}_{i}<1$. We find that the critical disorder strength for this to happen is much larger for correlated disorder than the corresponding value of $W / t$ for uncorrelated disorder.

In Fig. 4 we show the site occupation for a strongly interacting system $(U / t=8)$ with correlated disorder $(\xi=4)$. The disorder free system is in the $\mathcal{D}$ phase discussed above. With increasing $W / t$ the band insulating region at the center of the trap is depleted. At the same time, the Mott insulating regions are also slowly destroyed. To see more clearly the rate at which the band and Mott insulating regions are destroyed, we plot in Fig. 5 the number of consecutive sites $N_{\text {Mott }}$ and $N_{\text {Band }}$ such 


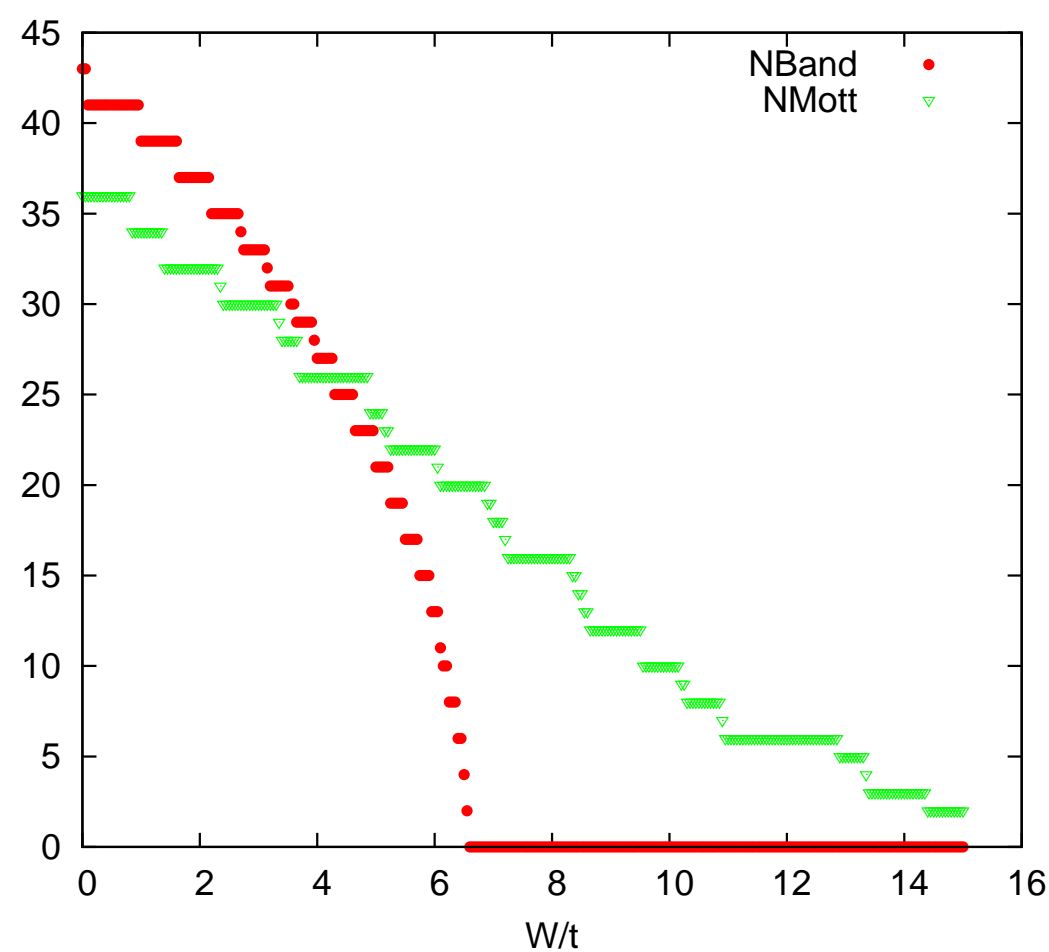

Fig. 5. (color online) The number of consecutive sites $N_{\text {Mott }}$ and $N_{\text {Band }}$ such that $\left|\mathcal{N}_{i}-1\right|<0$ and $\left|\mathcal{N}_{i}-2\right|<0$, respectively, as a function of $W / t$. The parameters are the same as in Fig. 4 .

that $\left|\mathcal{N}_{i}-1\right|<0$ and $\left|\mathcal{N}_{i}-2\right|<0$, respectively, as a function of $W / t$. Comparison with the same calculation in the uncorrelated disorder case 19 reveals the increased stability range of insulating regions for correlated disorder.

The effect of disorder on 1D fermions and in particular transitions between different phases can also be assessed through the thermodynamic properties. For this purpose we calculate the inverse compressibility defined as $\kappa^{-1}=\left\langle\left\langle N^{2} \delta \mu / \delta N\right\rangle\right\rangle_{\text {dis }}$ where $\mu$ is the chemical potential. In Fig. 6 we show $\kappa^{-1}$ as a function of $N$ for various values of the disorder correlation length $\xi$. The phase transitions can be identified as sharp kinks in a clean system $(W / t=0)$. Uncorrelated disorder has two main effects on $\kappa^{-1}$ as discussed in our previous work ${ }^{19}$ Firstly, the sharp features indicating phase transitions are smoothed out. Secondly, a large enhancement of $\kappa^{-1}$ at low density is observed. This is reminiscent of a similar behavior found in 2 D electron systems. As $N$ decreases the atoms mostly occupy the deepest valleys in the disorder landscape, thus the high density regions in the system tend to become disconnected. For a given interaction strength $u$ and low $N$ the system stiffens as disorder grows. In the present situation we observe that with increasing correlation length $\xi$ the low density enhancement in $\kappa^{-1}$ is diminished. The basic reason for 


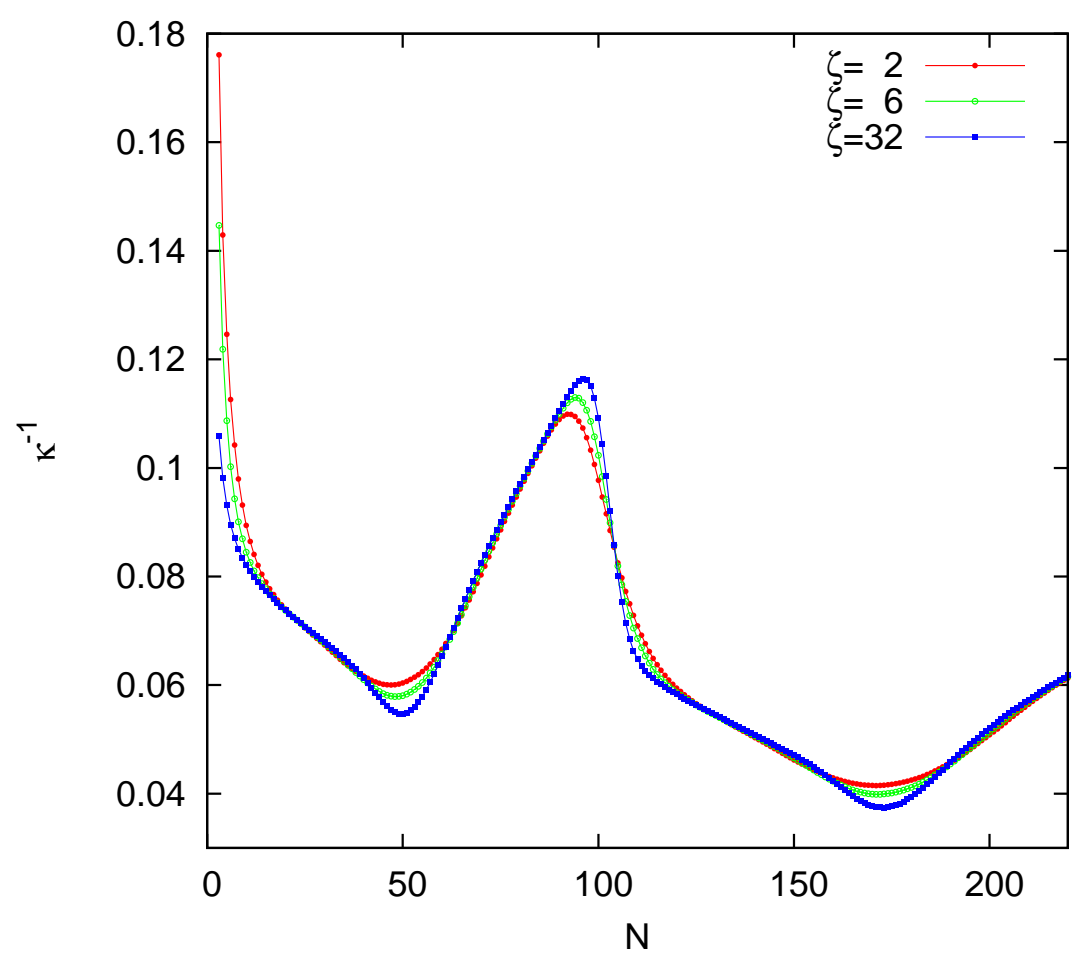

Fig. 6. (color online) The inverse compressibility $\kappa^{-1}$ (in units of $t$ ) in the presence of correlated disorder as a function of $N$ for $V_{2} / t=2.5 \times 10^{-3}, W / t=5, u=8$, and $L=200$ lattice sites. The correlation length $\xi=0$ (circles), $\xi=6$ (squares), and $\xi=32$ (triangles).

this behavior is that increasing $\xi$ makes the disorder potential more smooth and more shallow compared to the $\xi=0$ case. Therefore the atoms are less localized in a correlated disorder potential.

\section{Concluding remarks}

We have studied the one-dimensional Fermi-Hubbard model in a harmonic confinement potential and in the presence of correlated disorder. This is believed to represent cold fermionic atoms in an optical lattice created by standing laser waves. Our numerical calculations of the site occupations are based on a lattice version of density functional theory in which we make use of the exact solution of the one-dimensional Hubbard model to treat the exchange-correlation effects. Disorder affects the ground-state phases of the interacting Fermi gases confined in a harmonic potential and an optical lattice. The insulating regions appear to be stable against both uncorrelated and correlated disorder. The anomalous enhancement of the stiffness observed at low density for uncorrelated disorder decreases with increasing correlation length. We hope that our results on disorder effects will stimulate 
experimental investigations with cold Fermi atoms in the future.

\section{Acknowledgements}

G. X. was supported by NSF of China under Granr No. 10704066. B. T. acknowledges the support by TUBITAK (No. 106T052), TUBA and a travel grant from Julian Swchiwinger Foundation.

\section{References}

1. See for instance, E. Abrahams, S.V. Kravchenko, and M.P. Sarachik, Rev. Mod. Phys. 73, 251 (2001); B.L. Altshuler, D.L. Maslov, and V.M. Pudalov, Physica E 9, 209 (2001).

2. J.D. Reppy, J. Low Temp. Phys. 87, 205 (1992); B. Fåk, O. Plantevin, H.R. Glyde, and N. Mulders, Phys. Rev. Lett. 85, 3886 (2000), and references therein.

3. R. Fazio and H. van der Zant, Phys. Rep. 355, 235 (2001).

4. A. Punnoose and A.M. Finkel'stein, Science 310, 289 (2005); A.A. Shashkin, S. Anissimova, M.R. Sakr, S.V. Kravchenko, V.T. Dolgopolov, and T.M. Klapwijk, Phys. Rev. Lett. 96, 036403 (2006).

5. J.P. Eisenstein, L.N. Pfeiffer, and K.W. West, Phys. Rev. Lett. 68, 674 (1992); S. Ilani, A. Yacoby, D. Mahalu, and H. Shtrikman, ibid. 84, 3133 (2000); S.C. Dultz and H.W. Jiang, ibid. 84, 4689 (2000).

6. B. Tanatar, A.L. Subaşı, K. Esfarjani, and S.M. Fazeli, Int. J. Mod. Phys. B 21, 2134 (2007).

7. J.E. Lye, L. Fallani, M. Modugno, D.S. Wiersma, C. Fort, and M. Inguscio, Phys. Rev. Lett. 95, 070401 (2005); D. Clément, A.F. Varón, M. Hugbart, J.A. Retter, P. Bouyer, L. Sanchez-Palencia, D.M. Gangardt, G.V. Shlyapnikov, and A. Aspect, ibid. 95, 170409 (2005); C. Fort, L. Fallani, V. Guarrera, J.E. Lye, M. Modugno, D.S. Wiersma, and M. Inguscio, ibid. 95, 170410 (2005); for a review see V. Ahufinger, L. Sanchez-Palencia, A. Kantian, A. Sanpera, and M. Lewenstein, Phys. Rev. A 72, 063616 (2005).

8. J.I. Cirac and P. Zoller, Science 301, 176 (2003).

9. D. Clement, P. Bouyer, A. Aspect, and L. Sanchez-Palencia, Phys. Rev. A: 77, 033631 (2008); Y.P. Chen et al., e-print arXiv:0710.5187, to be published in Phys. Rev. A.

10. M. Greiner, O. Mandel, T. Esslinger, T.W. Hänsch, and I. Bloch, Nature 415, 39 (2002).

11. T. Schulte, S. Drenkelforth, J. Kruse, W. Ertmer, J. Arlt, K. Sacha, J. Zakrzewski, and M. Lewenstein, Phys. Rev. Lett. 95, 170411 (2005).

12. H. Moritz, T. Stöferle, K. Günter, M. Köhl, and T. Esslinger, Phys. Rev. Lett. 94, 210401 (2005).

13. W. Hofstetter, C.I. Cirac, P. Zoller, E. Demmler, and M.D. Lukin, Phys. Rev. Lett. 89, 220407 (2002); S. Trebst, U. Schollwök, M. Troyer, and P. Zoller, Phys. Rev. Lett. 96, $250402(2006)$.

14. I. Bloch, J. Dalibard, and W. Zwerger, arXiv:0704.3011v2, to be published in Rev. Mod. Phys.; L. Giorgini, L.P. Pitaevskii, and S. Stringari, e-print arXiv:0706.3360v2, to be published in Rev. Mod. Phys.

15. K. Schönhammer, O. Gunnarsson, and R.M. Noack, Phys. Rev. B 52, 2504 (1995); N.A. Lima, M.F. Silva, L.N. Oliveira, and K. Capelle, Phys. Rev. Lett. 90, 146402 (2003).

16. E.H. Lieb and F.Y. Wu, Phys. Rev. Lett. 20, 1445 (1968). 
November 20, 2018 0:2 WSPC/INSTRUCTION FILE 1dcorre

Effect of disorder on the interacting Fermi gases in a one-dimensional optical lattice 11

17. M. Rigol, A. Muramatsu, G.G. Batrouni, and R.T. Scalettar, Phys. Rev. Lett. 91, 130403 (2003); M. Rigol and A. Muramatsu, Phys. Rev. A 69, 053612 (2004); Opt. Commun. 243, 33 (2004); X.-J. Liu, P.D. Drummond, and H. Hu, Phys. Rev. Lett. 94, 136406 (2005); V.L. Campo, Jr. and K. Capelle, Phys. Rev. Lett. 72, 061602 (2005).

18. Gao Xianlong, M. Polini, M.P. Tosi, V.L. Campo, Jr., K. Capelle, and M. Rigol, Phys. Rev. B 73, 165120 (2006).

19. Gao Xianlong, M. Polini, B. Tanatar, and M.P. Tosi, Phys. Rev. B 73, 161103 (2006).

20. P.W. Anderson, Phys. Rev. 109, 1492 (1958).

21. J. Shi and X.C. Xie, Phys. Rev. Lett. 88, 086401 (2002).

22. K. Hirose, F. Zhou, and N.S. Wingreen, Phys. Rev. B 63, 075301 (2001); K. Hirose and N.S. Wingreen, ibid. 65, 193305 (2002); H. Jiang, D. Ullmo, W. Yang, and H.U. Baranger, ibid. 69, 235326 (2004); E. Räsänen and M. Aichinger, ibid. 72, 045352 (2005). 